\title{
Core-Collapse Supernovae as Dust Producers
}

\author{
Rubina Kotak \\ Astrophysics Research Centre, Queen's University Belfast, \\ Belfast, BT7 1NN, United Kingdom \\ email: r.kotak@qub.ac.uk
}

\begin{abstract}
Although it has long been hypothesised that core-collapse supernovae may produce large quantities of dust, interest in this problem has recently been rekindled given the enormous dust masses inferred at very high redshifts $(z \gtrsim 6)$, when conventional low-mass dust-producing stars would fail to contribute significantly to the universal dust budget. Emission due to warm dust peaks at mid-IR wavelengths. However, with the notable exception of SN 1987A, supernova studies in the mid-IR have been virtually non-existent until the advent of the Spitzer Space Telescope. On behalf of the Mid-Infrared Supernova Consortium, I briefly discuss recent exciting results from mid-IR studies of core-collapse supernovae using Spitzer and attempt to put the role of supernovae as major dust producers into perspective.
\end{abstract}

Keywords. supernovae: general - supernovae: individual (SN 2002hh, SN 2003gd, SN 2004dj, SN 2004et, SN 2005af) - dust, extinction - infrared: stars

\section{Introduction}

Recent years have witnessed a flurry of studies that have emphasised the important role that dust plays in our understanding of the near and distant Universe. Dust formation in the interstellar medium (ISM) has been shown to be extremely inefficient. The preferred sites for dust formation are the atmospheres of evolved, low-mass $\left(\mathrm{M}<8 M_{\odot}\right)$ stars from where it is transported into the ISM via stellar winds. This mechanism however, fails to explain the presence of dust at high redshifts as the evolutionary time-scales of these low-mass stars (up to $1 \mathrm{Gyr}$ ) begin to become comparable to the age of the Universe. Furthermore, the IR luminosities of $\mathrm{z}>6$ quasars (e.g. Bertoldi et al. 2003) imply enormous dust masses $\left(10^{8} M_{\odot}\right)$. The short time-scales required for dust enrichment make core-collapse supernovae rather natural candidates for dust producers in the early Universe.

It has long been hypothesized (Cernuschi et al. 1967, Hoyle \& Wickramasinghe, 1970, Tielens et al. 1990) that the physical conditions in the ejecta core-collapse supernovae may lead to the condensation of large amounts of dust. This stems from a combination of factors: (i) core-collapse supernova ejecta contain large amounts of refractory elements from which dust grains could form; (ii) cooling of the ejecta occurs by adiabatic expansion and, in some cases, by molecular emission; (iii) dynamical instabilities in the ejecta results in regions of enhanced density which may further aid the growth of grains by selfshielding. On the modelling side, there seems to be no apparent problem in producing substantial amounts $\left(0.1-1 M_{\odot}\right)$ of dust in supernova ejecta, even at high redshifts. This implies a dust condensation efficiency of about 0.2 (Morgan \& Edmunds, 2003).

Yet, the observational support for the hypothesis that grains condense in sufficient amounts in core-collapse supernovae is remarkably meagre, and recent claims are highly controversial. Two of the most compelling ways of detecting dust are: (i) the attenuation of spectral lines at optical/near-IR wavelengths in the nebular phase; (ii) thermal emission from dust grains. Until recently, the strongest evidence for dust formation in supernova 

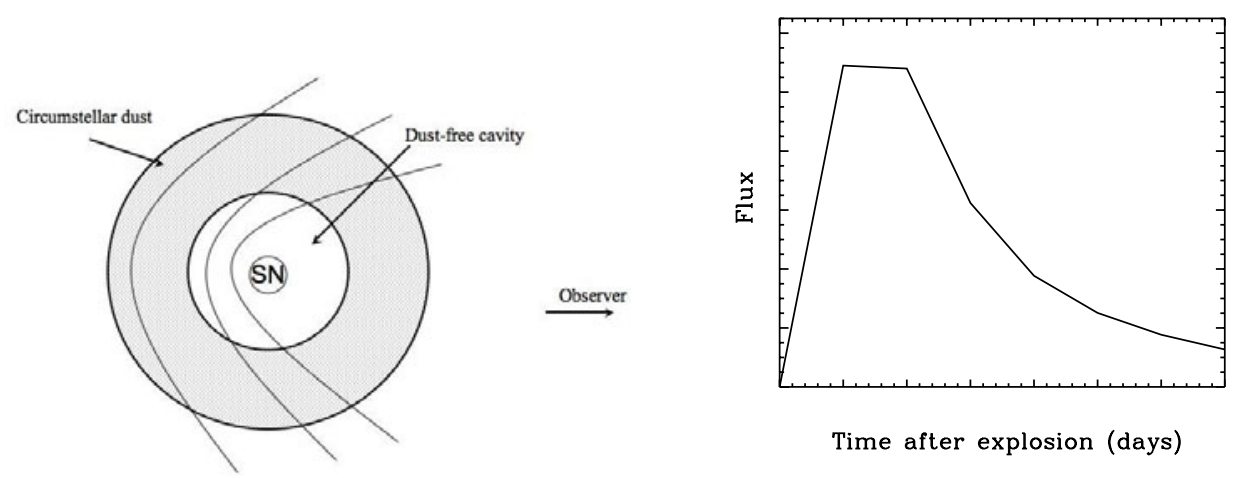

Figure 1. Left: Schematic illustration of an echo arising due to the explosion of a supernova in a dusty circumstellar medium. The series of paraboloids delineate the emitting volume which changes as a function of time. Adapted from Dwek (1983). Right: Example of a light curve resulting from a configuration such as that shown in the left-hand panel. Note the characteristic flat-top of the resulting light curve.

came from SN 1987A which showed a strong mid-IR excess that was accompanied by a decrease in optical emission and a blueward shift of emission line profiles (Lucy et al. 1989, Danziger et al. 1989, Wooden et al. 1991, Ercolano et al. 2007). However, even for this very well-studied object, the dust-mass estimates do not exceed $7.5 \times 10^{-4}$ (Ercolano et al. 2007).

While the attenuation of spectral lines at late times is a relatively unambiguous signature of the presence of dust, it is difficult to derive quantitative measures of the amount and nature of the dust. As warm grains emit most strongly in the mid-IR, this is the ideal wavelength range for following dust condensation in real time. However, groundbased mid-IR observations are challenging - if not unfeasible - for the vast majority of supernovae. Even for SN 1987A (at only $\sim 50 \mathrm{kpc}$ ), most of the mid-IR data came from the Kuiper Airborne Observatory (Wooden et al. 1991). Since the launch of the Spitzer Space Telescope, with vastly superior sensitivity and spatial resolution compared to previous instrumentation, this situation has been changing dramatically. With Spitzer, the Mid-Infrared Supernova Consortium (MISC) $\dagger$ has begun a vigorous programme of midIR supernova studies. Here, I will discuss recent exciting results, and attempt to put the role of core-collapse supernovae as dust producers into perspective.

\section{New dust or circumstellar dust?}

When studying the thermal emission from dust, it is important to bear in mind that even if a near- or mid-IR "excess" is detected, it might not necessarily be due to new dust that has condensed in the ejecta. Thermal emission may arise from pre-existing dust in the circumstellar medium e.g. due to a dusty wind from the progenitor star which has been heated by the flash from the supernova, resulting in an infrared echo (Bode \& Evans, 1979, Dwek, 1983). Shock heating due to ejecta-circumstellar matter interaction may be another mechanism which gives rise to an echo. A schematic diagram of a configuration that would give rise to an echo is shown in Fig. 1.

Clearly, an infrared echo could potentially mask any signature of newly condensing dust, given that the magnitude of this effect. However, in general, emission due echoes

$$
\dagger \text { http://star.pst.qub.ac.uk/ rk/misc.html }
$$




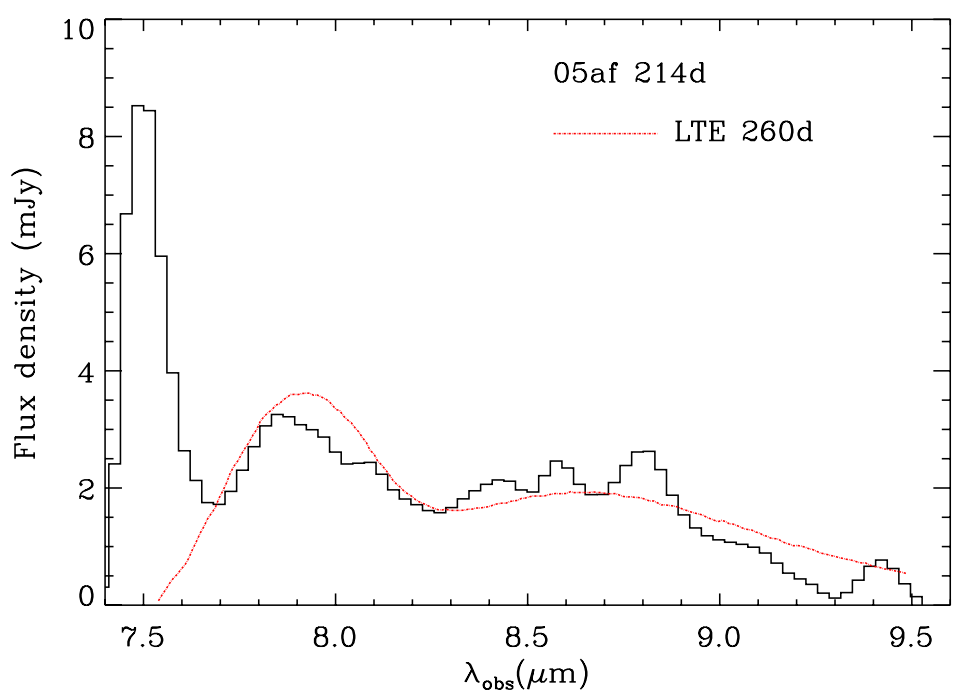

Figure 2. The $260 \mathrm{~d}$ LTE model from Liu \& Dalgarno (1994), attenuated by a factor of 2.5, superimposed on the continuum-subtracted second-epoch spectrum of SN 2005af. From this, we deduce a $\mathrm{SiO}$ mass of $\sim 2 \times 10^{-4} M_{\odot}$ (Kotak et al. 2006). The prominent line at $7.5 \mu \mathrm{m}$ is due to $[\mathrm{NiI}]$.

tends to appear earlier in the evolution of a supernova, compared to dust formation, which tends to occur at epochs of several hundred days. A caveat, of course, is the geometry and spatial extent of the circumstellar matter. Given the characteristic light curves that arise from echoes, one way of potentially distinguishing between pre-existing and new dust is to monitor the light curve to determine its shape (see Fig. 1). In situations where there is contribution to the infrared luminosity from both an echo, and new dust, the situation is complicated, and detailed modelling is required. Meikle et al. (2006) report the first detection of an IR echo in the most common of supernova types, the type-IIP supernova SN 2002hh. However, given the complexity of the field around the supernova, it was not possible to conclusively establish whether the echo was due to dusty pre-existing circumstellar matter, or a dusty molecular cloud.

\section{New mid-IR observations and models}

The first mid-IR spectrum of any supernova obtained since SN 1987A was that of the type II-P supernova, SN 2004dj which, at distance of $\sim 3 \mathrm{Mpc}$, was also the nearest since SN 1987A. The spectrum showed a strong red-wing of the CO fundamental band, and prominent lines of [NiI] and [NiII] (see Fig. 2 in Kotak et al. (2005)). The features described above appear in all of the type II-P supernovae in our sample. Another example is shown in Fig. 3. SN 2005af, also a type II-P supernova, showed the clear development of the $\sim 7.5 \mu \mathrm{m} \mathrm{SiO}$ feature from day 67 to day 214 (Fig. 2) - again, the first time since SN 1987A. Using the SiO model of Liu \& Dalgarno (1994), we estimate a SiO mass of $2 \times 10^{-4} M_{\odot}$.

The models shown in Fig. 3 are based on a spherical, uniform sphere of isothermal dust grains, following the escape probability treatment of Lucy et al. (1989). A typical grain size distribution is used for a mix of refractory materials as predicted by models of dust condensation in supernovae. In order to estimate the amount of dust, the mass 

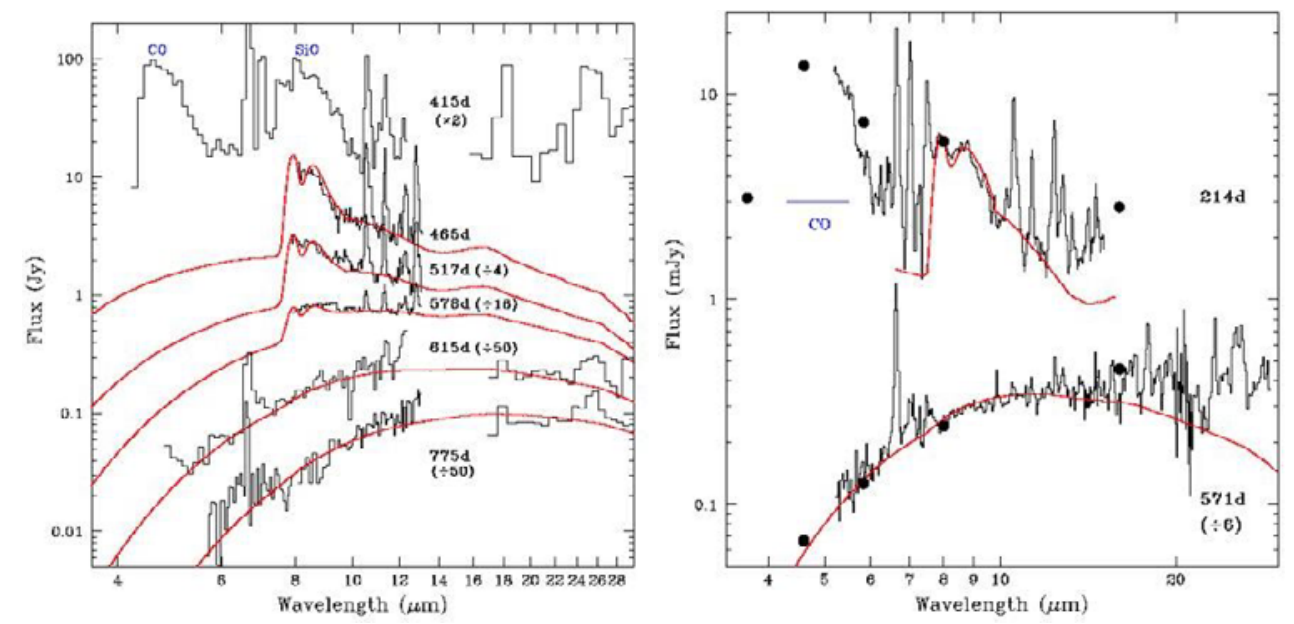

Figure 3. For both panels, the red lines show model fits to the data (see text) and include the SiO model of Liu \& Dalgarno (1994). Left panel: Mid-IR spectra of SN 1987A, taken from Roche et al. (1993) and Wooden et al. (1993). Right panel: Spectra of the type II-P supernova, SN 2005af. The day 214 spectrum is taken from Kotak et al. (2006). Filled circles: 5-16 $\mu \mathrm{m}$ photometry.

of dust is increased until an adequate match to the spectrum is obtained. In order to be conservative, we model the spectra for the most optically thin case that will still provide an adequate fit to the spectra (see Meikle et al. (2007) for details).

This approach was first tested on SN 1987A (Fig. 3), which yields a maximum mass of $\sim 6 \times 10^{-4} M_{\odot}$. This is consistent with estimates using other methods e.g. $7.5 \times 10^{-4} M_{\odot}$ (Ercolano et al. 2007). As with SN 2004dj, and SN 2005af, emission due to SiO and CO is clearly evident in SN 1987A. Also, a cool dust continuum provides a good match to the spectra. The same pattern holds for SN 2005af, with strong molecular emission at earlier epochs $(\sim 200 \mathrm{~d})$, which is replaced by a strong cool continuum at later times $(\sim 600 \mathrm{~d})$. Our dust mass estimate for SN 2005af comes to $\sim 4 \times 10^{-4} M_{\odot}$. In the latter spectrum, there is a hint of an even cooler component, which might increase this estimate somewhat.

Clearly, the estimates above represent the amount of directly detected dust. A key point to remember is that these are only firm lower limits - there may be substantially more dust present in optically-thick clumps. This problem was already identified in the context of SN 1987A (Lucy et al. 1989, Wooden et al. 1993). The problems persists even at wavelengths as long as $(24 \mu \mathrm{m}$, the extent of most of our data). In the event that clumps are optically-thick in the near-IR, but optically-thin in the mid-IR, it would be possible to obtain better constraints on the total dust mass by combining the data in both wavelength regions (Ercolano et al. 2007). However, for most - if not all - of our Spitzer targets, current indications are that the clumps are optically-thick in the mid-IR regime before significant dust condensation occurs.

Recently, Sugerman et al. (2006) claimed to have detected a significant amount of dust $\left(0.02 M_{\odot}\right)$ in the type II-P supernova, SN 2003gd. However, reanalysis of the same data by Meikle et al. (2007) led them to conclude that SN 2003gd was not special in any way compared to the rest of the mid-IR II-P sample. They were forced to this conclusion for a number of reasons: Firstly, the large dust mass reported by Sugerman et al. (2006) rests entirely on their $24 \mu \mathrm{m}$ flux measurement at an epoch of about $700 \mathrm{~d}$. However, based on numerous tests of the sensitivity of the Spitzer images at $24 \mu \mathrm{m}$ in the vicinity of the 
supernova, Meikle et al. (2007) showed that the Sugerman et al. (2006) flux is in error: it is too low by a factor of $\sim 4$. Secondly, their outer limit of their dust-forming zone lies at $8000 \mathrm{kms}^{-1}$, which is conflict with the observed metal line velocities of $\sim 2000 \mathrm{kms}^{-1}$ from late-time spectroscopy. Thus, the Sugerman et al. (2006) model implies a cooler dust component beyond $24 \mu \mathrm{m}$, for which there currently is no evidence. There is also a severe energy deficit in that the total radioactive decay energy deposited in the ejecta is a factor of $\sim 4$ too low to account for the observed luminosity. Even if one were to allow for a large uncertainty in the ${ }^{56} \mathrm{Ni}$ mass, the deficit would remain severe. Thus, on both energy and velocity grounds, most of the $24 \mu \mathrm{m}$ flux cannot be due to dust condensing in the ejecta.

\section{Summary}

From a sample of well-observed type II-P supernovae in the mid-IR, we find that all objects formed some dust. Prior to this work, evidence that the most common type of core-collapse supernova (the II-P subtype) form dust was meagre.

Grain formation models (e.g. Todini \& Ferrara, 2001, Nozawa et al. 2003) predict that carbon, silicate, and magnetite grains should be present in substantial quantities, with the silicate grains probably dominating. Our sample of core-collapse supernovae all show evidence of strong emission due to $\mathrm{CO}$, or $\mathrm{SiO}$, or both at epochs as early as $\sim 100 \mathrm{~d}$. Thus, although our sample size remains small, all of the supernovae that showed evidence for dust condensation, also showed evidence at earlier epochs of strong molecular emission. Thus, molecules may well play a crucial role in the dust formation process.

Current estimates of the amount of dust remain small: $10^{-3}$ to $10^{-5} M_{\odot}$, i.e., $10-100$ times lower than needed to account for the dust seen at high redshifts. However, the estimates presented here are only lower limits, and substantial amounts may well exist in optically-thick clumps.

Much work yet remains to be done in assessing the dust production as a function of supernova sub-type, and is limited mainly by current mid-IR facilities.

\section{References}

Bertoldi, F., Carilli, C. L., Cox, P., et al. 2003, A\&A, 406, L55

Blair, W. P., Ghavamian, P., Long, K. S., et al. 2007 ApJ, 662, 998

Bode, M. \& Evans, A. 1979, A\&A, 73, 113

Cernuschi, F., Marsicano, F., \& Codina, S. 1967, Ann. d'Astrophys, 30, 1039

Danziger, I. J., Gouiffes, C., Bouchet, P., \& Lucy, L. B. 1989, IAU Circ, 4746, 1

Dwek, E. 1983, ApJ, 274, 175

Ercolano, B., Barlow, M. J., \& Sugerman, B. E. K. 2007, MNRAS, 375, 753

Hoyle, F. \& Wickramasinghe, N. C. 1970, Nature, 226, 62

Kotak, R., Meikle, P., van Dyk, S. D., et al. 2005, ApJ, 628, L123

Kotak, R., Meikle, P., Pozzo, M., et al. 2006, ApJ, 651, L117

Liu, W. \& Dalgarno, A. 1994, ApJ, 428, L769

Lucy, L. B., Danziger, I. J., Gouiffes, C., \& Bouchet, P. 1989, in: G. Tenorio-Tagle, M. Moles \& J. Melnick (eds.), Structure and Dynamics of the Interstellar Medium (Berlin: SpringerVerlag), Proc. IAU Coll 120, 164

Meikle, W. P. S., Mattila, S., Gerardy, C. L., et al. 2006, ApJ, 649, 332

Meikle, W. P.S., Mattila, S., Pastorello, A., et al. 2007, ApJ, 665, 608

Morgan, H. L. \& Edmunds, M. G., 2003, MNRAS, 343, 427

Nozawa, T., Kozasa, T., Umeda, H., et al. 2003, ApJ, 598, 785

Roche, P. F., Aitken, D. K., \& Smith, C. H. 1993 MNRAS, 261, 522

Stanimirović, S., Bolatto, A. D., Sandstrom, K., et al. 2005, ApJ 632, L103 
Sugerman, B. E. K., Ercolano, B., Barlow, M. J., et al. 2006, Science, 313, 196

Tielens, A. G. G. M., 1990, in Carbon in the Galaxy: Studies from Earth and Space, NASA Conf. Publ., 3061, 59

Todini, P. \& Ferrara, A. 2001, MNRAS, 325, 726

Wooden, D. H., Rank, D. M., Bregman, J. D., et al. 1993, ApJS, 88, 477

\section{Discussion}

MoDJAZ: What about dust formation in supernova remnants?

Kotak: Supernova remnants may be net creators of dust, but also net destroyers of dust. Currently, there is evidence on both sides: e.g. Rho et al. (this conference) report about $0.02 M_{\odot}$ of dust in the Cassiopeia A supernova remnant, while Stanimirović et al. (2005) estimate in SNR 1E0102.2-7219 to be $\sim 8 \times 10^{-4} M_{\odot}$, and Blair et al. (2007) estimate $\sim 3-5 \times 10^{-4} M_{\odot}$ in the Kepler supernova remnant.

HiRschi: Do you expect dust formation in core-collapse supernovae to be metallicitydependent?

KoтAK: Yes, one would naively expect this to be the case. 